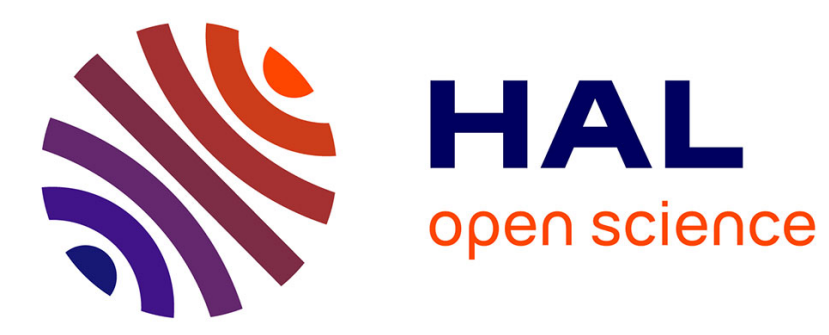

\title{
On the current distribution at the channel - rib scale in polymer-electrolyte fuel cells
}

\author{
Najib Belgacem, Joël Pauchet, Marc Prat
}

\section{To cite this version:}

Najib Belgacem, Joël Pauchet, Marc Prat. On the current distribution at the channel - rib scale in polymer-electrolyte fuel cells. International Journal of Hydrogen Energy, 2018, 43 (10), pp.5112-5123. 10.1016/j.ijhydene.2018.01.097 . hal-02001563

\section{HAL Id: hal-02001563 \\ https://hal.science/hal-02001563}

Submitted on 31 Jan 2019

HAL is a multi-disciplinary open access archive for the deposit and dissemination of scientific research documents, whether they are published or not. The documents may come from teaching and research institutions in France or abroad, or from public or private research centers.
L'archive ouverte pluridisciplinaire HAL, est destinée au dépôt et à la diffusion de documents scientifiques de niveau recherche, publiés ou non, émanant des établissements d'enseignement et de recherche français ou étrangers, des laboratoires publics ou privés. 


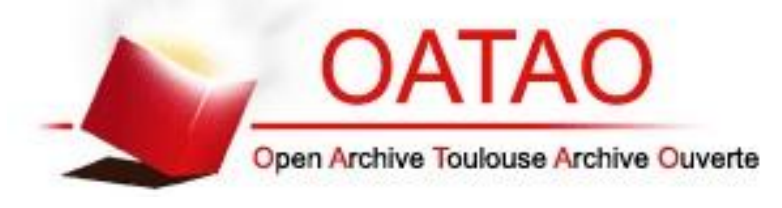

Open Archive Toulouse Archive Ouverte

OATAO is an open access repository that collects the work of Toulouse researchers and makes it freely available over the web where possible

This is an author's version published in: http://oatao.univ-toulouse.fr/21466

\section{Official URL:}

https://doi.org/10.1016/j.ijhydene.2018.01.097

\section{To cite this version:}

Belgacem, Najib and Pauchet, Joël and Prat, Marc On the current distribution at the channel e rib scale in polymerelectrolyte fuel cells. (2018) International Journal of Hydrogen Energy, 43. 5112-5123. ISSN 0360-3199

Any correspondence concerning this service should be sent to the repository administrator: tech-oatao@listes-diff.inp-toulouse.fr 


\title{
On the current distribution at the channel - rib scale in polymer-electrolyte fuel cells
}

\author{
Najib Belgacem ${ }^{a}$, Joel Pauchet ${ }^{b, * *}$, Marc Prat ${ }^{a, *}$ \\ a Institut de Mécanique des Fluides de Toulouse (IMFT), Université de Toulouse, CNRS, INPT, UPS, Toulouse, France \\ ${ }^{\mathrm{b}}$ Univ. Grenoble Alpes, CEA LITEN, DEHT, 17 Rue Martyrs, F-38054, Grenoble, France
}

Keywords:

Current density distribution

Pore network modelling

Condensation

Gas diffusion layer

Rib-channel

Continuum- PNM coupled model

\begin{abstract}
A B S T R A C T
Experimental results based on in-situ measurements at the interface between the catalyst layer and the gas diffusion layer (GDL) on the cathode side at the channel - rib scale show an interesting variation of the current density distribution as the mean current density is increased. It is found that the local current density below the rib median axis corresponds to a maximum at low to intermediate mean current densities and to a minimum when the mean current density is sufficiently high. Also, the higher is the current density, the more marked the minimum. From numerical simulations, it is shown that the current density distribution inversion phenomenon is strongly correlated to the liquid water zone development within the GDL.
\end{abstract}

\section{Introduction}

As pointed out in Ref. [1], the current density spatial distribution is of high interest in order to characterize the operation and performance of polymer electrolyte fuel cells (PEFCs). The fact that the current distribution is not uniform is well known, e.g. Ref. [1] and this can cause the loss of performance and contributes to degradation mechanisms leading to a reduced lifetime of the PEFCs [2]. Here, we focus on the current density distribution at the channel - rib scale. We first report experimental measurements showing an interesting variation in the current density distribution when the mean current density is varied. In particular, the measurements indicate a reduced current production under the rib when the mean current density is sufficiently high. This effect is not new. It has been reported in Ref. [3], also from local measurements. As in our experiments, the current density distributions reported in Ref. [3] show that the current density tends to become less and less uniform as the average current is increased and that the minimum current density tends to localize below the rib area. The existence of a minimum current density below the rib has been predicted from numerical simulations, e.g. Refs. [4-13]. Essentially, the phenomenon was explained by the oxygen starvation below the rib. However, the simulations reported in previous works, e.g. Refs.

\footnotetext{
* Corresponding author.

** Corresponding author.

E-mail addresses: joel.pauchet@cea.fr (J. Pauchet), mprat@imft.fr (M. Prat).
} 
[4-13], were all based on various questionable simplifications. In particular, the possible presence of liquid water in the gas diffusion layer (GDL) was completely ignored in the majority of those works. Exceptions are the works presented in Refs. $[5,6]$. However, the model used in Refs. [5,6] is isothermal and the presented results are actually not consistent with the existing measurements since the current density is predicted to be lower below the rib for any mean current density. On the other hand, the interpretation of the experimental results reported in Ref. [3] was not fully conclusive because the liquid water formation could not be detected. In other terms, the possible impact of the liquid water on the measured current density distributions could not be assessed.

As in previous attempts, e.g. Refs. [4-13], we rely on numerical simulations in order to better understand the changes in the structure of the current density distributions when the mean current density is varied. The significant difference is that we use a model taking into account both the temperature gradients within the GDL and the process of liquid water formation by condensation.

As discussed in Ref. [14], the modeling of transport phenomena in polymer-electrolyte fuel cells can be developed within various frameworks. The most classical one is the continuum approach to porous media in which each porous layer is seen as a fictitious continuum medium. As a recent example where this type of modelling is used, one can refer to [15]. However, as discussed in various articles, e.g. Refs. $[16,17]$ and references therein, the results obtained with the classical continuum approach can be questioned when the approach is applied to thin systems with only a few pores over the thickness, i.e. the GDLs, especially as regards the simulation of twophase flows. For such thin porous media, a potentially accurate option is to perform direct simulations, e.g. Ref. [18]. However, the computational time associated with this type of method is quite long and the computational domain often quite small. In between, we have the pore network models (PNM). PN modelling is a mesoscale approach based on a simplified representation of the pore space as a network of pores connected by narrower channels (also referred to as throats). The approach can be considered as a good trade-off. Much less computationally demanding than the direct simulation methods, the PNM approach does not suffer from the shortcomings of the continuum approach. In particular, PNMs are well adapted to simulate the capillary-fingering flow regime commonly considered in the modelling of two-phase flows in PEMFC, e.g. Ref. [19]. This explains why PNM is now a somewhat popular approach for modelling two-phase flows in GDL, e.g. Refs. $[17,20,21]$, where numerous references are given. Whereas the study of two-phase flows in GDL using PNM has been often performed in the past without explicit coupling with the other transport phenomena, it can be noted that this coupling is taken into account in more recent works, e.g. Refs. $[17,20,22,23]$. Actually, the idea in those works is to combine the continuum approach where it is appropriate, i.e. for the catalyst layer (CL), the microporous layers (MPL) and the membrane, with pore network modelling for the layers where PNM is clearly more appropriate, i.e. the fibrous layer of the GDLs. In line with this state of the art, we use for the simulations in the present paper the coupled continuum PNM model described in Ref. [20]. This model of a PEMFC cathode couples the electro-chemical phenomena taking place in the catalyst layer with a pore network model (PNM) for computing the transfers and the liquid water formation in the fibrous diffusion medium (DM) of the GDL and a continuum approach in the MPL. As emphasized in Ref. [20], distinguishing features of this PNM are to assume that the water forming in the CL enters the GDL in vapor form and to model the liquid water formation by condensation in the DM.

Actually, it was already shown in Ref. [20] that the current density distribution predicted by the model was different under dry condition (no liquid water in GDL) and wet condition (liquid water in GDL) with the localization of the minimum current density below the rib for the wet conditions, thus qualitatively as in the existing measurements. However, the current density distribution was shown for only one mean current density for the wet condition. Here, the current density distributions will be presented over a much larger range of mean current densities so as to establish a much clearer correlation between the liquid water formation and the change in the current density distribution. Also, no experimental results on the current density distributions were presented in Ref. [20].

The article is organized as follows. The experimental results of interest are briefly presented in the next section. Then some basic features of the modelling are recalled. Main results of simulations are then presented both as regards the current density distribution and the liquid water distribution. A discussion is proposed before the conclusion.

\section{Experiments}

The measurement method is along the same lines as the one described in Ref. [3]. It is based on a reverse method exploiting the measurement of electrical potential in the membrane electrode assembly (MEA) core with thin tungsten wires of $25 \mu \mathrm{m}$ placed at $115 \mu \mathrm{m}$ from each other at the interface of the catalyst layer and the microporous layer. One can refer to [24] for a detailed description of the experimental set-up and measurement methods. It is important to note that those measurements were performed at a fuel cell standard operating temperature of $80^{\circ} \mathrm{C}$. Here also it was not possible from the experiments to relate the current density minimum localization with the possible impact of liquid formation. The main results obtained in Ref. [24] and of interest for the present study are shown in Fig. 1.

As can be seen from Fig. 1, the transverse current densities distribution varies with both the relative humidity $(R H)$ in the channel and the mean current density $\bar{i}$. For the three $R H$ tested, the transverse current density is uniform over the CLGDL interface for a sufficiently low mean current density (approximately $\bar{i}<0.2 \mathrm{~A} / \mathrm{cm}^{2}$ ). Then, as the mean current density is increased, the local current density tends to be slightly higher in the region of the CL-GDL interface located below the rib than below the channel (this corresponds to mean current densities approximately in the range $\left.\left[0.2-0.6 \mathrm{~A} / \mathrm{cm}^{2}\right]\right)$. This trend is reversed in the range of the highest mean current densities $\left(\bar{i}>0.6 \mathrm{~A} / \mathrm{cm}^{2}\right)$. The local current density is then significantly lower in the region of the interface located below the rib. As can be seen also from Fig. 1, the non-uniformity of 


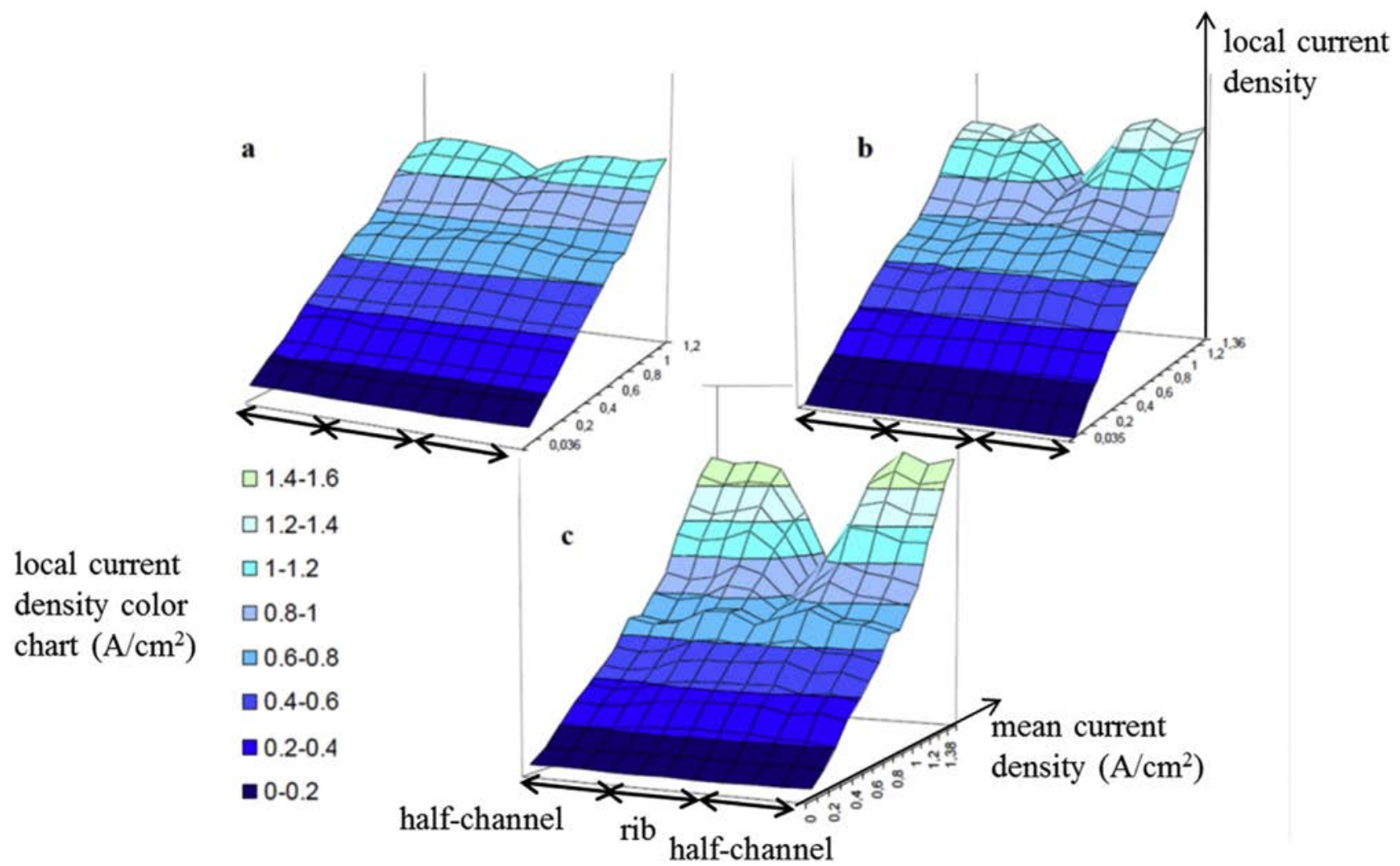

Fig. 1 - Measured transverse current density distributions as a function of mean current density at the CL/MPL interface at the rib-channel scale reported in Ref. [24] for three values of the relative humidity in the channel: $\mathrm{a}$ ) $\mathrm{RH}=30 \%, \mathrm{~b}) \mathrm{RH}=50 \%$, c) $\mathrm{RH}=80 \%, \mathrm{P}=1.5 \mathrm{bar}, \mathrm{T}=80^{\circ} \mathrm{C}, \mathrm{P}_{\mathrm{O} 2} / \mathrm{P}=21 \%$.

the local current density at high mean current density significantly increases as $\mathrm{RH}$ is increased. The difference in local current density between the regions below the channels and the region below the centre line of the rib can be as high as about $0.5 \mathrm{~A} / \mathrm{cm}^{2}$ for the highest tested mean current density $\left(\bar{i} \approx 1.35 \mathrm{~A} / \mathrm{cm}^{2}\right)$.

\section{Modelling}

As mentioned in the introduction, we use the computational model presented in Ref. [20]. In this model, the GDL is represented by a $52 \times 52 \times 7$ cubic pore network (the figures indicate the number of pores along the directions of a Cartesian grid), see Ref. [20] for more details. The computational domain is depicted in Fig. 2. It corresponds to an elementary unit cell containing a bipolar plate rib and two gas half channels as for the experimental domain. The geometrical dimensions of the various layers are specified in Fig. 2. For the GDL, the indicated thickness corresponds to the uncompressed GDL, i.e. below the channels.

As sketched in Fig. 2, the GDL is considered as a two-layer porous system, made of a fibrous diffusion medium (DM) and a MPL coated onto the DM.

It should be noted that the results shown in Fig. 1 were obtained with a GDL from Freudenberg (H2315T10AC1) whereas the parameters in the model are specified so as to represent a SGL 25BC (the model was developed within the framework of a project using this type of GDL). We believe that the studied problem, i.e. the impact of liquid water formation on local current distribution, is generic and therefore can be studied, at least qualitatively, using the parameters corresponding to SGL 25BC.

The properties of the various layers are specified as explained in Ref. [20]. There are summarized in Tables 1 and 2 in the appendix for the MPL and the DM, respectively. As can be seen from the appendix, the highly anisotropic behavior of the DM is taken into account as well as the fact the DM is compressed below the rib. The properties of the other layers, namely the catalyst layer and the membrane are given in Ref. [20].

\section{Computed local current density distributions}

Examples of current density distributions at the CL-GDL interface obtained from the simulations are depicted in Fig. 3. As can be seen, there is clear change in the position of the current intensity maximum when the mean current density is increased. Consider for example the color maps for $\mathrm{RH}=90 \%$ in Fig. 3 and look at the line corresponding to the rib median axis (this corresponds to the vertical dashed median line in 


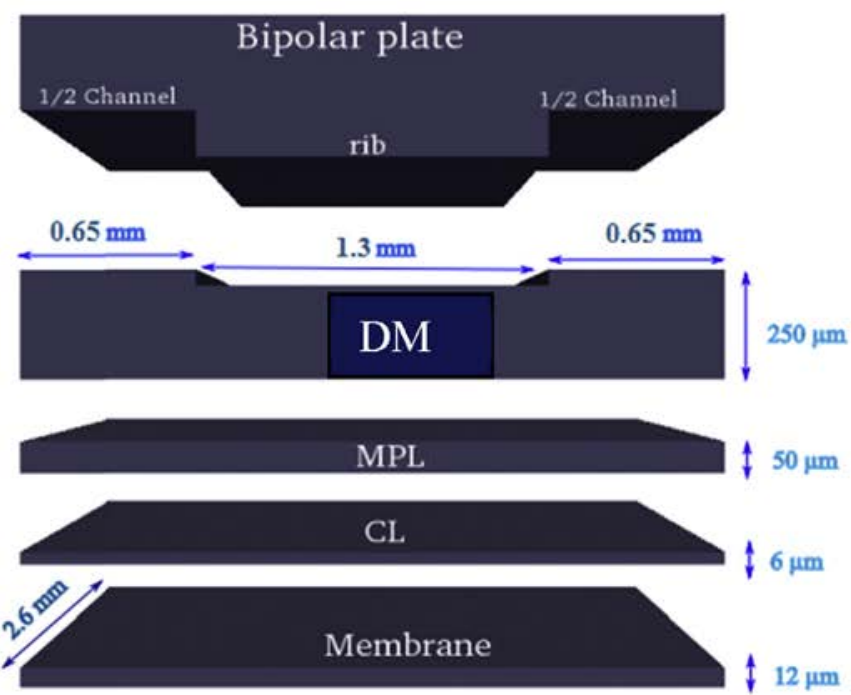

a)

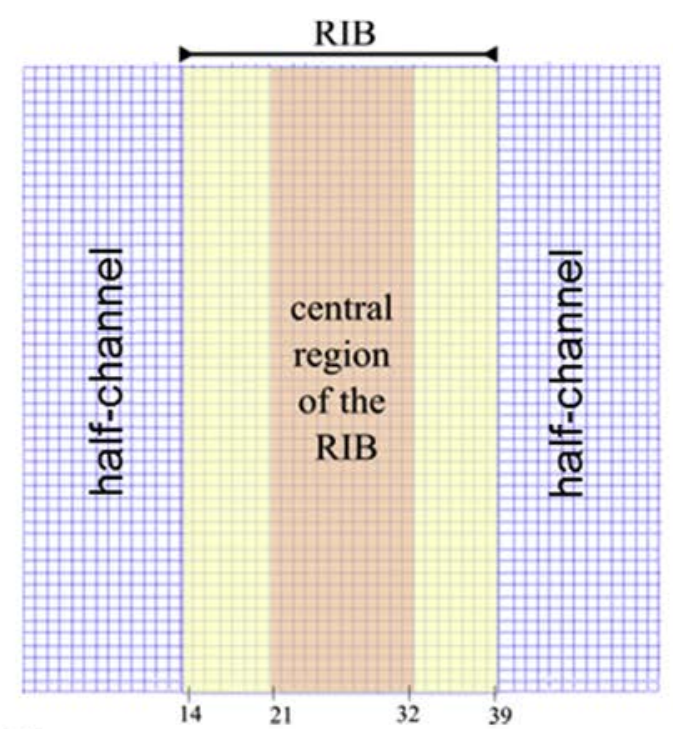

b)

Fig. 2 - a) Exploded view of computational domain, b) definition of central region of the rib (the figures correspond to pore rows in the transverse direction).

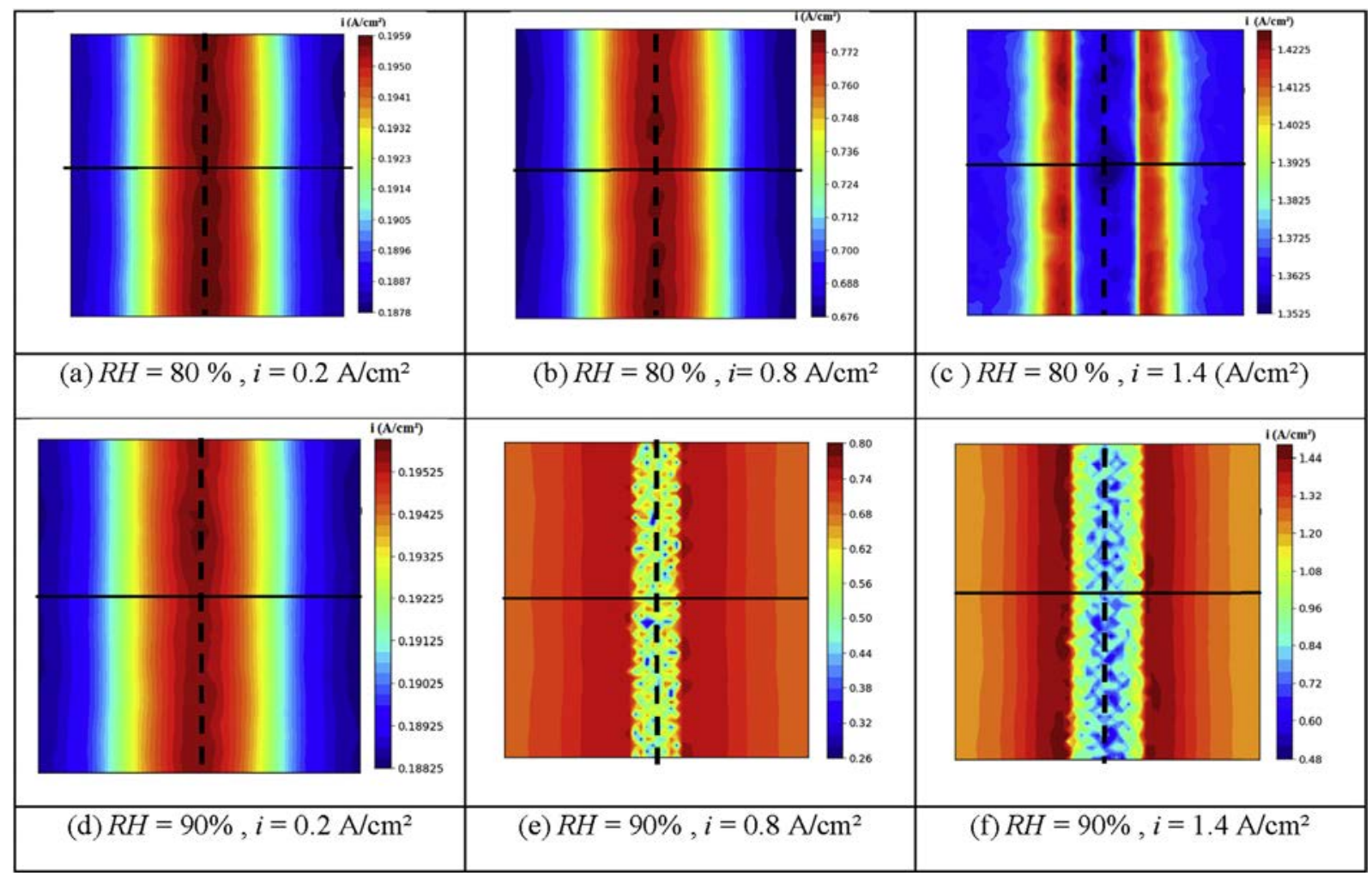

Fig. 3 - Impact of mean current density on the current density distribution at the CL/GDL interface for $\mathrm{RH}=80 \%$ (top row) and $90 \%$ (bottom row).

Fig. 3). This line corresponds to a maximum local current density for $\bar{i}=0.2 \mathrm{~A} / \mathrm{cm}^{2}$ whereas it corresponds to a minimum local current density for $\bar{i}=0.8$ and $\bar{i}=1.4 \mathrm{~A} / \mathrm{cm}^{2}$. Similarly, in the case of $\mathrm{RH}=80 \%$, the rib median axis corresponds to a maximum local current density at $\bar{i}=0.2$ and 0.8 $\mathrm{A} / \mathrm{cm}^{2}$ but to a minimum at $\bar{i}=1.4 \mathrm{~A} / \mathrm{cm}^{2}$.
Still more interesting are the computed local current density transverse profiles at channel - rib scale (at the CL-GDL interface) depicted in Fig. 4. The profiles correspond to the local current density distributions along the horizontal black solid line visible in the middle of each colored panel in Fig. 3. The profiles for $\mathrm{RH}=80 \%$ clearly show that the local current 


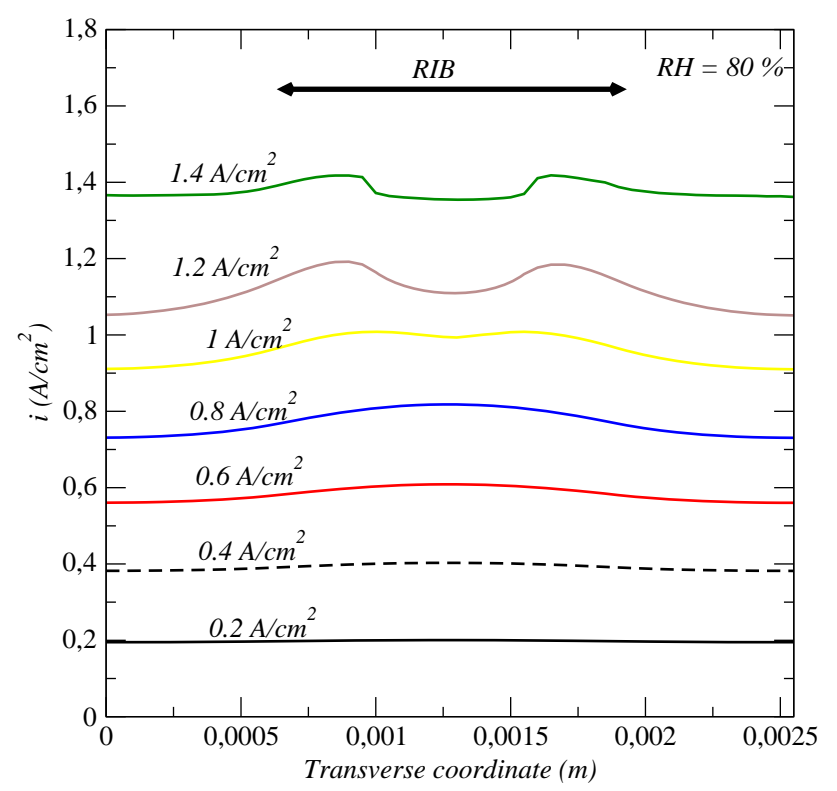

a)

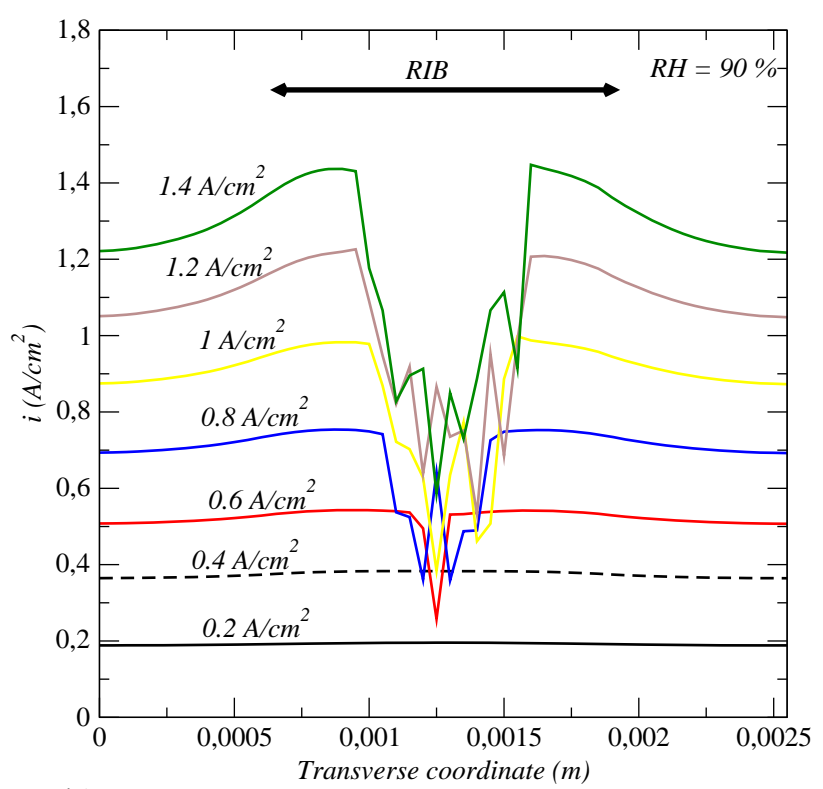

b)

Fig. 4 - Current density transverse profiles at the CL - GDL interface for various mean current densities: a) $R H=80 \%$, b) RH $=90 \%$.

density is uniform between rib and channel for low mean current densities $\left(<0.2 \mathrm{~A} / \mathrm{cm}^{2}\right)$. Then, a maximum of local current density appears below the rib as the mean current density is increased. For higher mean current density ( 1 A/ $\mathrm{cm}^{2}$ ), the local current density below the rib is not a maximum anymore and can even be a well-marked minimum for a higher relative humidity in the channel ( $R H=90 \%$, Fig. $4 b)$. The greater is the mean current density, the more marked the minimum.

These profiles are in a good qualitative agreement with the experimental profiles depicted in Fig. 1. Note the local maximum in current density along the edges of the rib (rib/ channel transition region) in Fig. 4 for the highest mean current densities. Although less clearly, this is also visible in Fig. 1. The computed amplitudes of the local current density variations are also consistent with the experimental measurements.

\section{Computed liquid water distributions}

\section{Condensation diagram}

As introduced in Ref. [21], the condensation diagram gives the operating conditions $(\bar{i}, R H)$ leading to a dry GDL, i.e. with no liquid water in the GDL, or a wet GDL, i.e. a GDL with liquid water formed by condensation assuming that the other parameters (operating temperature, pressure, etc) are set constant and thus do not vary. The method to compute this diagram is described in Ref. [21]. The condensation diagram obtained for the specified values of model parameters is shown in Fig. 5. The line in this figure marks the boundary

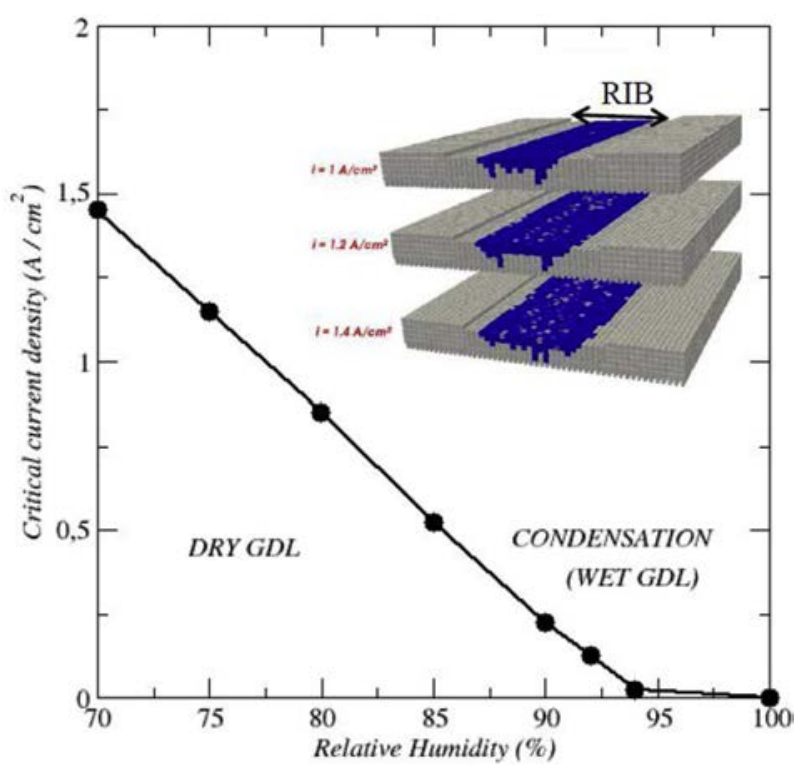

Fig. 5 - Example of condensation diagram. The inset shows typical computed liquid water distribution (in blue) within the DM resulting from the condensation process for three mean current densities, $\mathrm{RH}=80 \%, \mathrm{~T}=80^{\circ} \mathrm{C}, \mathrm{P}_{\mathrm{O} 2} / \mathrm{P}=21 \%$. (For interpretation of the references to color in this figure legend, the reader is referred to the Web version of this article.)

between the "dry" operating conditions and the "wet" boundary conditions and corresponds to the curve $\bar{i}_{c}(R H)$, where $\bar{i}_{c}$ is, for a given $R H$, the "critical" current density, i.e. the minimum mean current density leading to condensation. 
Interestingly, the concept of critical current density marking the transition from a dry GDL to a wet GDL was already introduced in Ref. [25], where it is referred to as the threshold current density. However, it should be noted that condensation and temperature spatial variations were ignored in Ref. [25] where furthermore a somewhat standard continuum model, thus also in the DM, was used.

In relation with the experimental results shown in Fig. 1, this diagram indicates that liquid water due to condensation is present in the DM for a sufficiently high mean current density $\left(\sim 0.8 \mathrm{~A} / \mathrm{cm}^{2}\right.$ for $\left.\mathrm{RH}=80 \%\right)$.

\section{Liquid water distribution}

The inset in Fig. 5 shows typical computed liquid water distributions within the DM resulting from the condensation process. This figure illustrates that the liquid water preferentially forms in the region below the rib, which corresponds to the region of lower temperatures within the DM. The preferential formation of liquid water in the rib area is in agreement with in situ experimental observations, e.g. Refs. $[26,27]$. The fact that the liquid water is mostly below the rib and the regions of the DM below the channels are free of liquid water is referred to as the separation effect [21].

As illustrated in Fig. 5 and expected from a physical standpoint since the water production rate increases with the current density, the region occupied by liquid water below the rib within the DM increases when the mean current density is increased. Fig. 5 also shows that the overall liquid saturation increases when the relative humidity is greater in the channel. These two features, i.e. the increase in saturation when either the mean current density or the channel relative humidity is increased, are also in a good qualitative agreement with in-situ experiments [26,27].

\section{Saturation profiles}

The liquid distribution in the DM is also illustrated in Fig. 6 showing the plots of the through plane and transverse inplane saturation profiles.

The transverse in-plane saturation is the fraction of the pore space occupied by liquid water in a longitudinal vertical slice (see inset in Fig. 6a). There are 52 pores in the transverse direction and therefore 52 longitudinal vertical slices. Therefore the in -plane saturation is computed in each of the 52 vertical slices. Similarly, the through plane saturation is the fraction of the pore space occupied by liquid water in a horizontal slice (see inset in Fig. 6a). There are six rows of pores to represent the DM in the through-plane direction. Therefore, the through plane saturation profile is formed by the saturation computed in each of these six rows. In the plots of the through plane saturation profile, the MPL is on the left side (pore 0 ) and the interface with the channel/rib area is on the right side (pore 6).

The saturation profiles obtained from the simulations for the case $\mathrm{RH}=80 \%$ are shown in Fig. $6 \mathrm{a}-\mathrm{b}$. As can be seen and consistently with the condensation diagram depicted in Fig. 5, there is no liquid water forming in the DM when the mean current density is lower than $1 \mathrm{~A} / \mathrm{cm}^{2}$ in Fig. $6 \mathrm{a}-\mathrm{b}$. The overall saturation increases with the current density (for $\bar{i}>0.8 \mathrm{~A} / \mathrm{cm}^{2}$ in Fig. $6 \mathrm{a}-\mathrm{b}$ ) but the liquid water remains confined below the rib area.

As can be seen from Fig. $6 c-d$ and also from the condensation diagram (Fig. 5), liquid water begins to form for a much lower mean current density when the relative humidity in the channel is increased (from $80 \%$ in Fig. $4 a-b$ to $90 \%$ in Fig. $6 c-d)$. This is in agreement with in-situ experimental observations, e.g. Ref. [28]. Consistently, the overall saturation is greater for a given mean current density when the channel relative humidity is increased (as can be seen from the comparison between the profiles for $\mathrm{RH}=80 \%$ and $\mathrm{RH}=90 \%$ in Fig. 6). Here again, the liquid water remains confined below the rib region area, even for the greatest considered mean current density $\left(1.4 \mathrm{~A} / \mathrm{cm}^{2}\right)$.

\section{Correlation between the change in the current density distribution and the liquid water formation}

The comparison between the current density profiles in Fig. 4 and the saturation profiles in Fig. 6 for $\mathrm{RH}=80 \%$ and $\mathrm{RH}=90 \%$ clearly shows a direct correlation between the formation of the current density minimum below the rib and the formation of liquid water due to condensation. For the case $\mathrm{RH}=80 \%$, there is no liquid water for $\bar{i}<1 \mathrm{~A} / \mathrm{cm}^{2}$. For this range of current density $\left(\left[0-0.8 \mathrm{~A} / \mathrm{cm}^{2}\right]\right)$, Fig. 4 indicates a maximum in current density in the region corresponding to the rib median axis. A similar maximum is also visible in the experimental results for the sufficiently low mean current densities. By contrast, the region corresponds to a local minimum in current density in the range $\left(\left[1-1.4 \mathrm{~A} / \mathrm{cm}^{2}\right]\right)$ when there is a sufficient amount of liquid water in the GDL. For $\mathrm{RH}=90 \%$, liquid water is present for each considered mean current density. However, it can be seen from Fig. $6 c-d$ that there is a significantly greater amount of liquid water in the range of mean current densities $\left[0.6-1.4 \mathrm{~A} / \mathrm{cm}^{2}\right]$. This is the range where the current density minimum in the region of the rib median axis is increasingly more marked as the mean current density increases.

The current density distribution inversion, i.e. the fact that the current density below the rib median axis rather corresponds to a local current density maximum at low mean current densities and to a minimum when the mean current density is sufficiently high, can be quantitatively illustrated from the computation of $\delta i$ defined as

$\delta i=\frac{1}{12} \sum_{j=n_{\min }}^{j=n_{\max }}(i(j)-\bar{i})$

where $i(j)$ is the current density along the current density transverse profile located in the row \#j counted from the left side of the GDL unit cell. The transverse profiles depicted in Fig. 4 are constructed from 52 discrete values corresponding to the number of pores (52) in the transverse direction. Then $n_{\min }$ (=21) and $n_{\max }(=32)$ corresponds to the central region of the rib (the width of the rib counted in number of pores is 26 , the rib extents from the rows \#14 to \#39, the rows 21 to 32 thus 


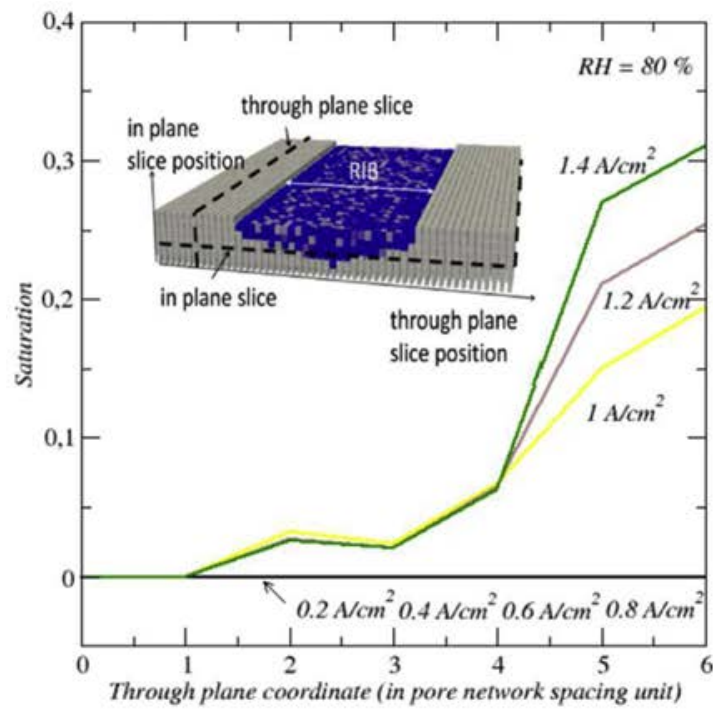

a) through plane

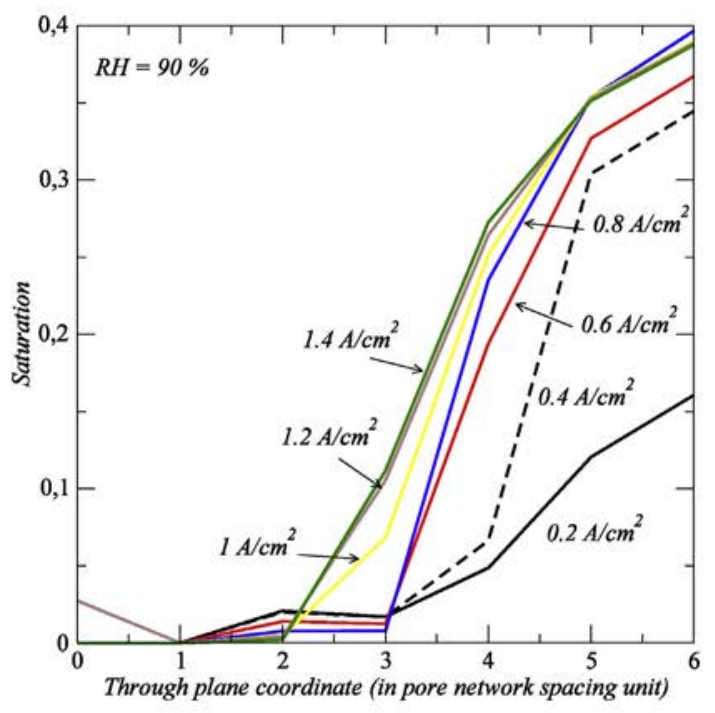

c) through plane

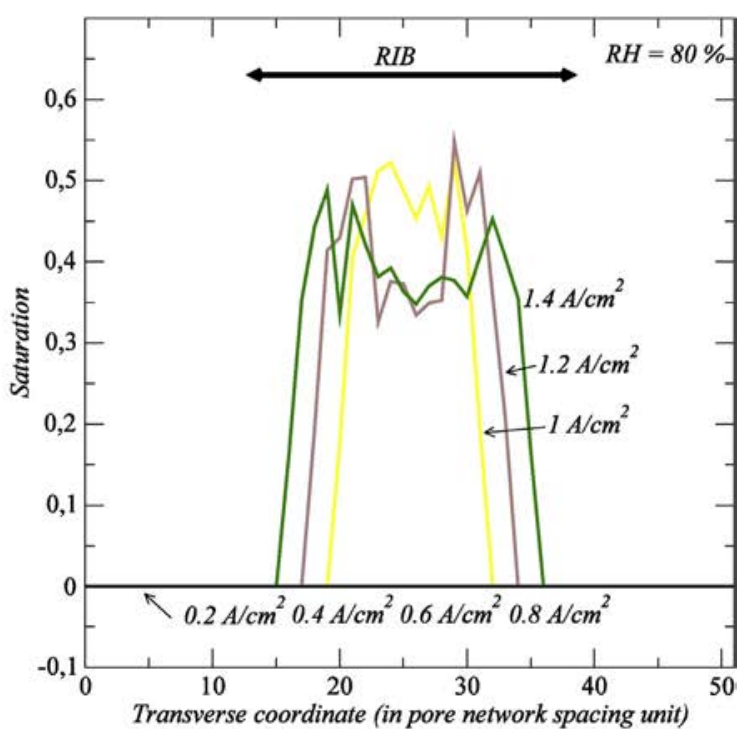

b) in plane

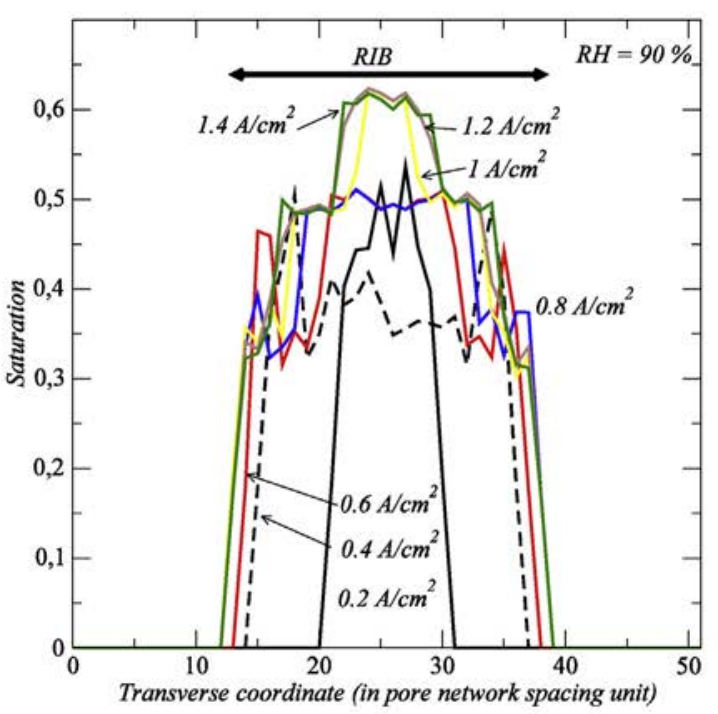

d) in plane

Fig. 6 - Impact of mean current density on saturation profiles for $R H=80 \%$ (top row) and $R H=90 \%$ (bottom row): a) c) through plane saturation profiles (MPL on the left, rib-channel on the right), b) d) transverse in plane saturation profiles. The in plane and through plane slices in the DM over which the saturation is computed are sketched in the inset in Fig. $6 a$.

correspond to the central region of the rib as illustrated in Fig. 2b). Thus $\delta$ i corresponds to the mean deviation between the current density in the central region of the rib and the mean current density. $\delta \mathrm{i}>0$ corresponds to the existence of a local maximum in the rib central region whereas $\delta i<0$ corresponds to a local minimum.

The computation of $\delta i$ leads to the results depicted in Fig. 7. They clearly illustrate that the current density distribution inversion below the rib region can be directly interpreted as a consequence of the growth of the zone occupied by liquid water in the region of the DM below the rib. In addition, it is clear that the inversion appears for lower current densities and is more marked for $\mathrm{RH}=90 \%$ than for $\mathrm{RH}=80 \%$.

\section{Discussions}

\section{Oxygen distribution}

As in previous works, e.g. Ref. [3] and references therein, the main obvious idea to explain the results discussed in the previous section, i.e. the current density distribution inversion below the rib, is to consider that the development of the liquid region below the rib affects the oxygen access to the region of the catalyst layer located below the central region of the rib as well as the distribution of water vapor in this region.

As illustrated in the inset in Fig. 5, the growth of the liquid zone in the DM reduces the fraction of the pore space available 


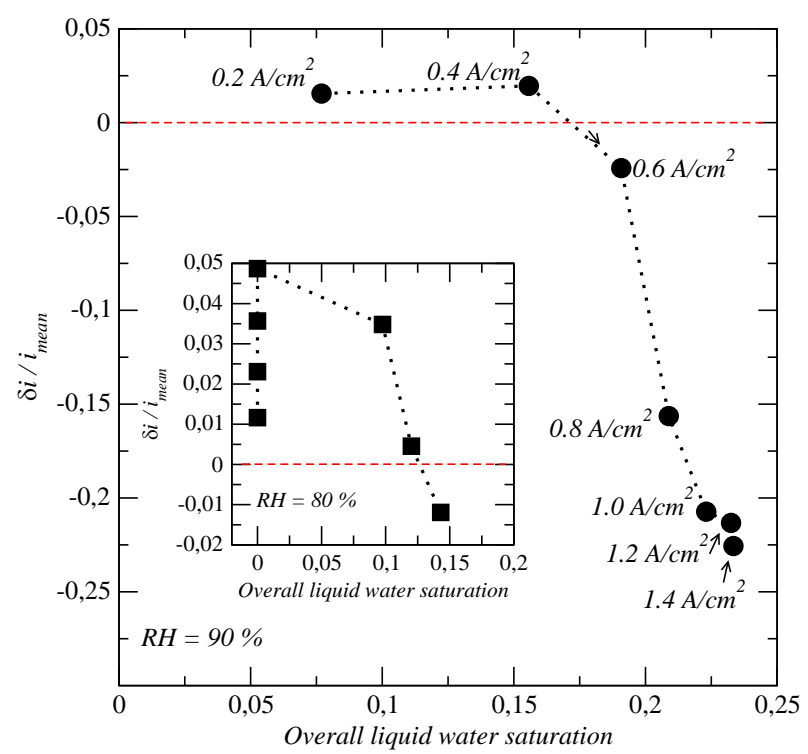

Fig. 7 - Current density mean deviation in the central region of the rib as a function of the overall liquid saturation; the black squares in the inset $(\mathrm{RH}=80 \%)$ correspond to the same mean current densities as the black dots $(\mathrm{RH}=90 \%)$.

for the transport of the oxygen in this region. The consequence is a decrease in the oxygen partial pressure at the CL MPL interface in this region as the liquid zone grows. This is illustrated in Fig. 8, which shows that the effect is quite marked. The transverse distribution in oxygen is only slightly non-uniform at low mean current densities. The fact that the oxygen partial pressure is minimum below the rib for the lower mean current density is then attributed to the longer diffusion path between the channel and the central region of the rib compared to the region of the CL-GDL interface located below the channel. This longer path induces a higher diffusion resistance and thus a smaller oxygen concentration below the middle of the rib even when the local current density is uniform between rib and channel. The oxygen partial pressure minimum becomes much more marked compared to the region below the channel when the liquid water zone develops below the rib.

\section{Water vapor distribution}

The trend is exactly the opposite regarding the water vapor partial pressure.

As illustrated in Fig. 8, the central region below the rib corresponds to a water vapor partial pressure maximum. This maximum is due to the longer diffusion path from this region to the channel when the DM is dry (sufficiently low current densities). The maximum is much more marked when liquid water forms in the DM below the rib. As the liquid water zone develops, the water vapor partial pressure at the CL-GDL interface below the rib central region tends to get closer to the saturation water vapor pressure since menisci progressively get closer to this interface.
This explains the change in the curves shape in Fig. $8 \mathrm{c}$ (which corresponds to the case $\mathrm{RH}=90 \%$ and thus to an important development of the liquid water zone), from a dome to a hat profile shape. This change is correlated to the change in the transverse saturation profile depicted in Fig. 6 . These results can thus be seen as another illustration of the strong impact of liquid water distribution on gas diffusion [29].

\section{On the link between the water vapor, oxygen and current} density distributions

However, the link with the local current density variations depicted in Fig. 4 is less straightforward than one might think at first glance.

As presented in Ref. [20], our model relies on the ButlerVolmer equation $[30,31]$, which provides the relationship between the local current density $i$ and the overpotential $\eta$ :

$i=i_{0}\left(\exp \left(\frac{\alpha n F}{R T} \eta\right)-\exp \left(-\frac{(1-\alpha) n F}{R T} \eta\right)\right)$

where $R=8.3 \mathrm{~J} /(\mathrm{mol} . \mathrm{K})$ is the ideal gas constant, $T$ is the temperature, $F=96485.3 \mathrm{~A} / \mathrm{mol}$ is the Faraday constant and $n=2$ ( $n$ is the number of chemical species involved in the reaction).

In Eq. (2), the exchange current density $i_{0}$ is expressed as:

$i_{0}=n k_{0} \exp \left(-\frac{A_{0}}{R T}\right)\left(a_{\mathrm{O}_{2}}^{\gamma \mathrm{O}_{2}}\right)^{1-\alpha}\left(a_{\mathrm{H}_{2} \mathrm{O}}^{\gamma_{\mathrm{H}} \mathrm{O}}\right)^{\alpha}$

in which $k_{0}=4.2 .10^{-8} \mathrm{~m} . \mathrm{s}^{-1}, \mathrm{~A}_{0}=24360 \mathrm{~J} \cdot \mathrm{mol}^{-1}, \alpha=0.6$, $\gamma_{\mathrm{O}_{2}}=0.41, \gamma_{\mathrm{H}_{2} \mathrm{O}}=2.04$ (as discussed in Ref. [20]). In Eq. (3), $a_{\mathrm{O}_{2}}=\frac{P_{\mathrm{O}_{2}}}{P_{\text {ref }}}, \quad a_{\mathrm{H}_{2} \mathrm{O}}=\frac{P_{\mathrm{H}_{2} \mathrm{O}}}{P_{\text {sat }}}$ are the oxygen and water activities respectively $\left(\mathrm{P}_{\mathrm{O}_{2}}\right.$ and $\mathrm{P}_{\mathrm{H}_{2} \mathrm{O}}$ are the oxygen and water vapor partial pressures in the gas phase within the catalyst layer).

Eq. (3), and thus also Eq. (2), are therefore directly dependent on the composition of the gas phase in oxygen and water vapor at the CL - GDL interface.

It can be seen from Eq. (3) that the current density decreases with a decreasing oxygen partial pressure. However it increases with an increasing water vapor partial pressure. Thus we cannot readily conclude from Fig. 8 that $i_{0}$ decreases below the rib as a result of liquid zone growth. Computing $i_{0}$ from the data depicted in Fig. 8 does lead to the expected behavior, i.e. a local minimum in $i_{0}$ forms below the central region of the rib for a sufficiently high mean current density. This indicates that the impact of the liquid zone growth on the distribution of $\mathrm{P}_{\mathrm{O} 2}$ has more impact on $i_{0}$ than the distribution of $P_{\mathrm{H} 2 \mathrm{O}}$ (which would tend to lead to a local maximum in $i_{0}$ and not a local minimum). However, the computed amplitude of the $i_{0}$ variations is not sufficient to fully explain the results plotted in Fig. 4.

Returning to Eq. (2), this is an indication that the dominant factor in the transverse variation of $i$ is the transverse variation in the overpotential $\eta$ (the temperature varies only slightly along the CL-GDL interface). In fact, in our model we first determine the electronic potential $\psi_{c}$ which is related to the overpotential $\eta$ through the series of equations (see Ref. [20]): 


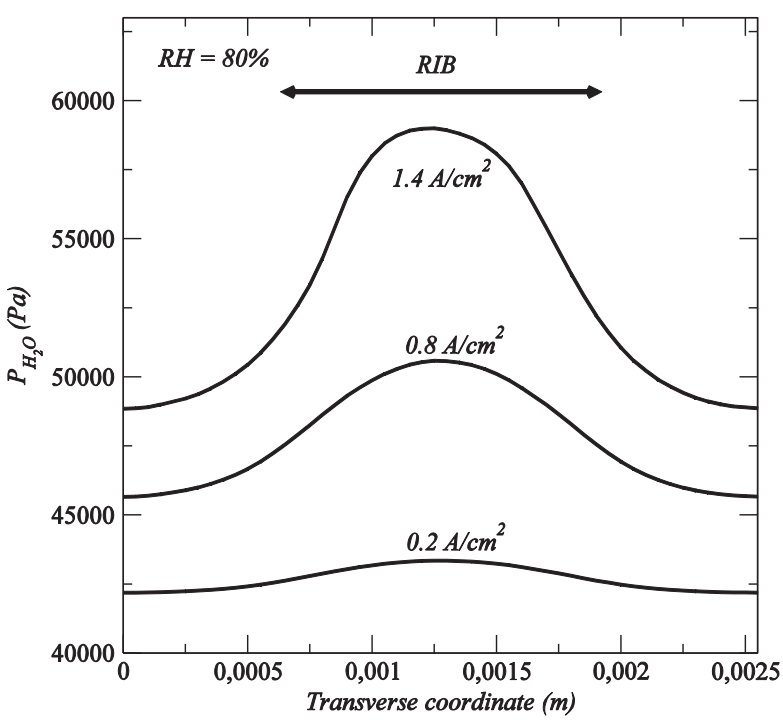

a)

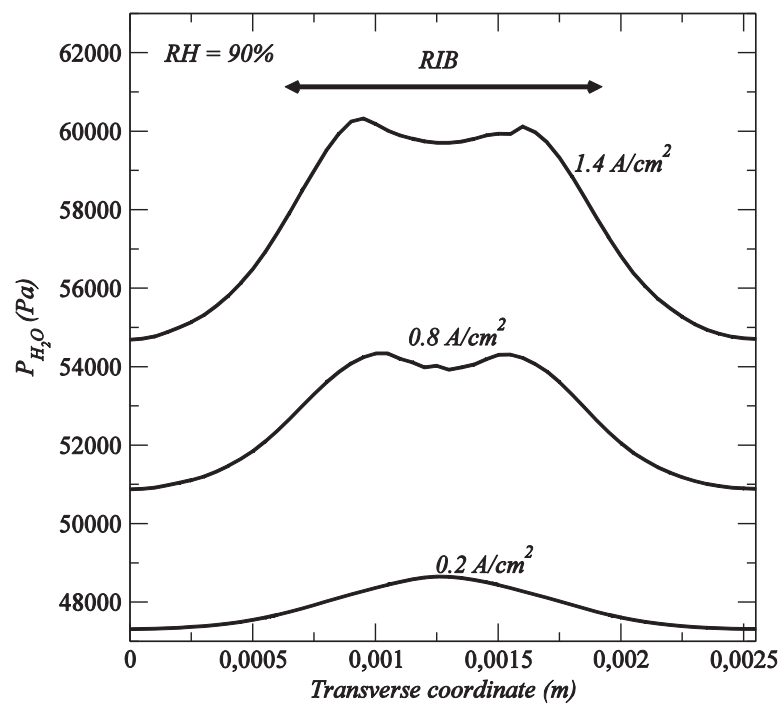

c)

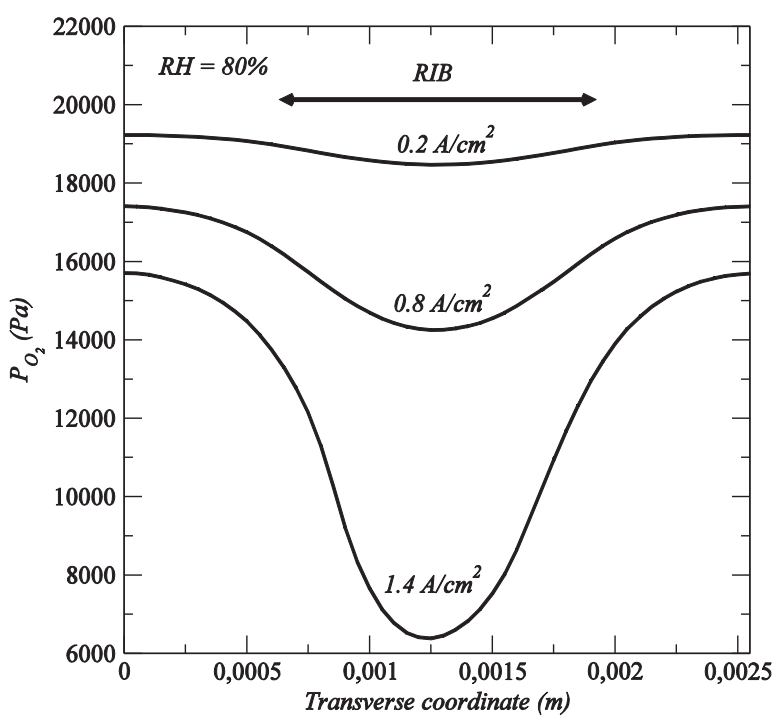

b)

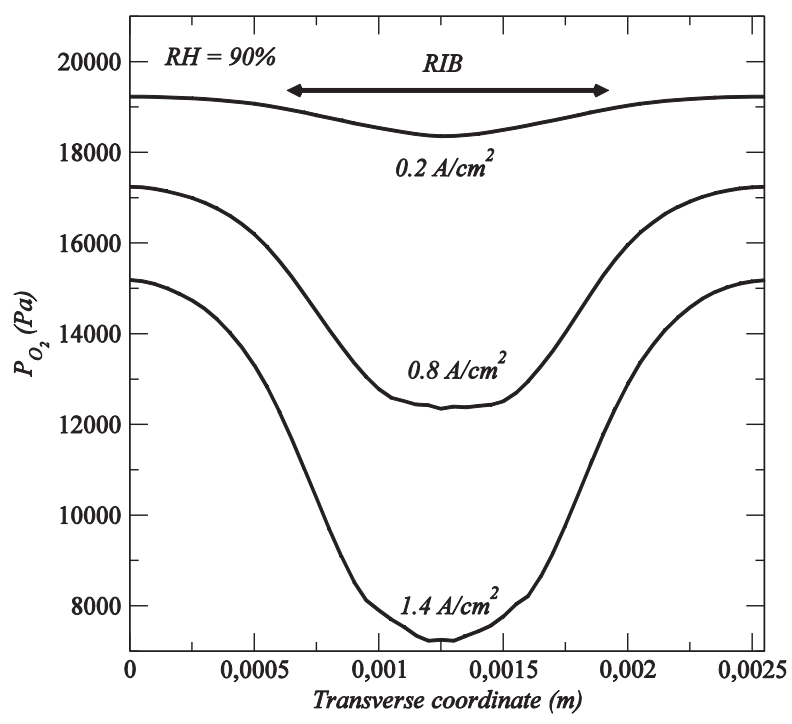

d)

Fig. 8 - Water vapor and oxygen partial pressure transverse profiles at the CL - GDL interface for various mean current densities: a) b) $R H=80 \%$, c) d) $R H=90 \%$.

$$
\left\{\begin{array}{c}
E_{\text {rev }}=\frac{\Delta H-T \Delta S}{n F}+\frac{R T}{n F} \ln \left(a_{\mathrm{O}_{2}}^{\frac{1}{2}} a_{\mathrm{H}_{2}} a_{\mathrm{H}_{2} \mathrm{O}}^{-1}\right) \\
\psi_{c}=E_{\text {rev }}+\eta+\phi_{c} \\
\eta=-\frac{R T}{(1-\alpha) n \mathrm{~F}} \ln \left(-\frac{\mathrm{i}}{\mathrm{i}_{0}}\right) \\
\phi_{c}=\phi_{a}+R_{m} \mathrm{i}
\end{array}\right.
$$

where it turns out that the thermodynamic potential $E_{\text {rev }}$ $(\sim 1.22 \mathrm{~V})$ is dominated by the first term $\left(\frac{\Delta H-T \Delta S}{n \mathrm{~F}}\right)$ and thus varies quite weakly with the activities $a_{\mathrm{O} 2}$ and $a_{\mathrm{H} 20}$. Also it can be noted that the membrane resistance $R_{m}$ is on the order of 5 $10^{-6} \Omega / \mathrm{m}^{2}$ for a well hydrated membrane. Thus the term $R_{m} i$ in Eq. (4) is on the order of $0.05 \mathrm{~V}(50 \mathrm{mV})$.
The simulations indicate that the electric potential $\psi_{c}$ varies little in the transverse direction when there is no liquid water formation $\left(\bar{i}=0.2 \mathrm{~A} / \mathrm{cm}^{2}\right)$ whereas the variation is noticeable in the presence of a liquid zone $(R H=90 \% \bar{i}=0.8 \mathrm{~A} /$ $\mathrm{cm}^{2}$ ) with a traverse variation of the order of $30 \mathrm{mV}$ and a complete change in the profile (local maximum in $\psi_{c}$ below the central region of the rib in the presence of liquid water whereas a local minimum is observed when the DM is dry).

Also the analysis of the two contributions to $\psi_{c}$, namely $\eta$ and $R_{m} i$ (note that $\phi_{a}=0$ by convention) shows that there are both of the same order of magnitude and vary like $\psi_{c}$. Thus, this confirms that the greater source of transverse variation in Eq. (2) is $\eta$ (and not $i_{0}$ ). Since $\eta, R_{m} i$ and $i$ are coupled through Eqs. (2)-(4), this, however, do not provide a fully direct explanation. Nevertheless, since it is clear from Eqs. (2)-(4) 
that the solution to electronic transport problem (i.e. i, $\psi_{c}$ ) depends of the activities of $\mathrm{O}_{2}$ and $\mathrm{H}_{2} \mathrm{O}$, we conclude that combination of the activities variations due to the liquid zone growth and the coupling between the various transport phenomena expressed in part by Eqs. (2)-(4) lead to the observed current inversion effect.

\section{Summary}

Our conclusion is therefore that the observed current density inversion effect as the mean current density is increased is essentially due to the development of the condensation liquid water zone below the rib. It can be argued, however, that the effect is observed for somewhat higher values of the relative humidity in the channel compared to the experimental results depicted in Fig. 1. As mentioned before, the condensation diagram for our model (Fig. 5) does not exactly correspond to the experimental situation. This is because we did no try to impose in our model the parameters corresponding to the experiments but simply used the values of parameters as specified in Ref. [20]. Trends are so strikingly similar between the experiments and the simulations that it is expected that using parameters a bit more representative of the experiments would simply still improve the agreement between the simulations and the experiments.

\section{On the liquid water formation in the GDL}

It is well known that water management is a key issue in PEMFC technology and still largely an open issue. As pointed out in many previous works, e.g. Refs. [18,27,32-34], understanding the exact mechanisms of water formation and transport into the membrane electrode assembly is therefore a crucial point for the PEMFC technology. Let's consider the gas diffusion layer (GDL) on the cathode side. Literature reviews [20,21], show that two quite different mechanisms of liquid water formation in the GDL have been considered so far, namely i) formation of liquid water resulting from the capillarity controlled invasion in liquid phase at the interface between the GDL and the catalyst layer (CL), e.g. Refs. [35,36], ii) condensation of the water vapor within the GDL, e.g. Refs. [20,21]. Water enters the GDL from the CL in vapor phase in the latter case whereas it enters the GDL in liquid phase in the first scenario. As mentioned in the previous sections, the pore network simulations of the condensation scenario are in agreement, at least qualitatively, with several experimental in - situ observations of liquid water distribution in GDL, e.g. Refs. [26-28]. This strongly suggests that the condensation of water vapor is a major mechanism of liquid water formation in the GDL. However, this is for a mean temperature of $80^{\circ} \mathrm{C}$. Although this temperature is considered as a typical operating temperature in many applications, lower operating temperatures are also possible. Experimental observations at significantly lower temperatures $\left(\sim 30-40{ }^{\circ} \mathrm{C}\right)$ [37] indicate significantly different liquid water distribution in the GDL. Thus, it is likely that a transition occurs in the mechanisms leading to the liquid water formation in the cathode GDL as the mean temperature is varied. As a result, the two aforementioned scenarios, liquid invasion and condensation, are likely not to be contradictory. This crucial point is currently somewhat unclear and would certainly deserve further studies. Whatever the situation, it is interesting to confirm the relevance of the condensation scenario for the upper range of temperature, i.e. close to $80^{\circ} \mathrm{C}$, since this range corresponds to common operating conditions.

In this respect, the favorable comparison between the experiments and the simulations regarding the current density distributions can thus be viewed as an additional confirmation that condensation is a major mechanism of liquid water formation in GDL in a fuel cell operating under standard conditions $\left(\mathrm{T} \sim 70-80^{\circ} \mathrm{C}\right)$. This is so not only as regards the water management, i.e. the improvement of performance, but also as regards another crucial point: the degradation of performance $[38,39]$. As illustrated in the present article, the transport phenomena are fully coupled in a PEM fuel cell. As a result, the degradation mechanisms also depend on the coupled character of the transfers, e.g. Ref. [40]. Therefore, any improvement in the understanding of water transfer is also of importance for the analysis of degradation mechanisms.

Although we conclude that the present study can be also seen as an additional confirmation of the relevance of the model presented in Ref. [20], it can be however noted that the experiments, e.g. Ref. [27], indicate that liquid water can be present almost everywhere within the GDL, thus not only in the region below the rib, for the standard operating condition $\left(\sim 80^{\circ} \mathrm{C}\right)$ when the relative humidity is very high $(\sim 100 \%)$ in the channel. The model must be tested and probably extended to deal with this situation, which is not rare in PEFC operation. This situation is, however, beyond the scope of the present paper since no data on the current density distributions were presented in Ref. [24] for fully humidified conditions.

\section{Conclusions}

Consistently with previous works, experimental in-situ measurements on the CL/GDL interface on the cathode side of a PEMFC show that the local current density is not uniform at the channel - rib scale. The local current density is higher below the rib than below the channel for low mean current densities and much lower for higher current densities.

A model [20] was used to simulate and analyze the experiments, and compute the local current density distributions for different mean current densities and relative humidity in the cathode feeding channels. A key feature of this model is to simulate the process of liquid water formation due to condensation in the diffusive medium thanks to a pore network model using a recently proposed condensation algorithm [21].

The simulation results are in very good qualitative agreement with the experimental results. As in the experiments, the simulation shows the inversion of the current density distribution in the region of the diffusion medium below the rib when the mean current density is varied. The local current density profile between rib and channel is rather uniform for low mean current densities. Then the local current density maximum forms below the rib for intermediate current densities whereas this region becomes the locus of the local current density minimum for higher current densities. As the relative humidity increases in the channel, the minimum below the rib is more marked and appears for lower current densities. The 
simulations indicate that this variation in the current density distribution is directly correlated to the development of the condensation liquid water zone in the GDL below the rib.

The favorable comparison between the experiments and the simulations also sheds light on the water transfer mechanisms. It confirms that condensation is a major mechanism of liquid water formation in GDL in a fuel cell operating -under standard conditions $\left(\mathrm{T} \sim 70-80^{\circ} \mathrm{C}\right)$. The case of the fully humidified conditions in the channel remains, however, to be explored since the model has not yet been tested for those conditions, which lead to liquid water distributions significantly different from the ones obtained for lower values of the relative humidity, [27].

As pointed out by one of the reviewers of the original version of the present paper, it would be interesting to study the influence of other operating conditions, such as oxygen fraction, pressure, and temperature for instance in addition to the influence of relative humidity and total current density as presented in this paper. This would allow obtaining a more comprehensive assessment of the factors affecting the performance in relationship with local current density distribution, gas diffusion and liquid water management.

\section{Acknowledgements}

This research has received funding from the European Union's Seventh Framework Programme (FP7/2007-2013) for the Fuel Cells and Hydrogen Joint Technology Initiative under grant agreement no 30 3452, "IMPACT - Improved Lifetime of Automotive Application Fuel Cells with ultra-low Pt-loading".

\section{Appendix}


[13] Meng H, Wang CY. Electron transport in PEFCs. J Electrochem Soc 2004;151:A358-67.

[14] Weber AZ, Borup RL, Darling RM, Das PK, Dursh TJ, Gu W, et al. A critical review of modeling transport phenomena in polymer-electrolyte fuel cells. J Electrochem Soc 2014;161:F1254-99.

[15] García-Salaberri PA, Sánchez DG, Boillat P, Vera M, Friedrich KA. Hydration and dehydration cycles in polymer electrolyte fuel cells operated with wet anode and dry cathode feed: a neutron imaging and modeling study. J Power Sources 2017;359(15):634-55.

[16] Rebai M, Prat M. Scale effect and two-phase flow in a thin hydrophobic porous layer. Application to water transport in gas diffusion layers of PEM fuel cells. J Power Sources 2009;192:534-43.

[17] Aghighi M, Hoeh MA, Lehnert W, Merle G, Gostick J. Simulation of a full fuel cell membrane electrode assembly using pore network modeling. J Electrochem Soc 2016;163:F384-92.

[18] Hao L, Cheng P. Lattice Boltzmann simulations of water transport in gas diffusion layer of a polymer electrolyte membrane fuel cell. J Power Sources 2010;195:3870-81.

[19] Sinha PK, Wang CY. Pore-network modeling of liquid water transport in gas diffusion layer of a polymer electrolyte fuel cell. Electrochim Acta 2007;52(28):7936-45.

[20] Belgacem N, Prat M, Pauchet J. Coupled continuum and condensation-evaporation pore network model of the cathode in polymer-electrolyte fuel cell. Int J Hydrogen Energy 2017;42:8150-65.

[21] Straubhaar B, Pauchet J, Prat M. Pore network modelling of condensation in gas diffusion layers of proton exchange membrane fuel cells. Int J Heat Mass Transf 2016;102(1):891-901.

[22] Zenyuk IV, Medici EF, Allen JS, Weber AZ. Coupling continuum and pore-network models for polymerelectrolyte fuel cells. Int J Hydrogen Energy 2015; $40: 16831-45$.

[23] Medici EF, Zenyuk IV, Parkinson DY, Weber AZ, Allen JS. Understanding water transport in polymer electrolyte fuel cells using coupled continuum and pore-network models. Fuel Cells 2016;16:725-33.

[24] Rachidi S. Développement et exploitation d'une microinstrumentation pour les piles à combustible de type PEMFC [unpublished Ph.D. thesis]. France: Poitiers University; 2011.

[25] Wang ZH, Wang CY, Chen KS. Two-phase flow and transport in the air cathode of proton exchange membrane fuel cells. J Power Sources 2001;94:40-50.

[26] Oberholzer P, Boillat P. Local characterization of PEFCs by differential cells: systematic variations of current and asymmetric relative humidity. J Electrochem Soc 2014;161(1):F139-52.
[27] Eller J, Roth J, Marone F, Stampanoni M, Büchi FN. Operando properties of gas diffusion layers: saturation and liquid permeability. J Electrochem Soc 2017;164:F115-26.

[28] LaManna JM, Chakraborty S, Gagliardo JJ, Mench MM. Isolation of transport mechanisms in PEFCs using high resolution neutron imaging. Int J Hydrogen Energy 2014;39:3387-96.

[29] García-Salaberri PA, Hwang G, Vera M, Weber AZ, Gostick JT. Effective diffusivity in partially-saturated carbon-fiber gas diffusion layers: effect of through-plane saturation distribution. Int J Heat Mass Transf 2015;86:319-33.

[30] Erdey-Gruz T, Volmer M. Zur theorie der wasserstoffüberspannung. Z Phys Chem A 1930;150:203-13.

[31] Mann RF,Amphlett JC, Peppley BA, Thurgood CP. Application of Butler-Volmer equations in the modelling of activation polarization for PEM fuel cells. J Power Sources 2006;161:775-81.

[32] Dai W, Wang H, Yuan XZ, Martin JJ, Yang D, Qiao J, et al. A review on water balance in the membrane electrode assembly of proton exchange membrane fuel cells. Int J Hydrogen Energy 2009;34:9461-78.

[33] Banerjee R, Kandlikar SG. Two-phase flow and thermal transients in proton exchange membrane fuel cells-A critical review. Int J Hydrogen Energy 2015;40:3990-4010.

[34] Chevalier S, Gea N, Georgea MG, Lee J, Banerjee R, Liua H, et al. Synchrotron $\mathrm{X}$-ray radiography as a highly precise and accurate method for measuring the spatial distribution of liquid Water in operating Polymer Electrolyte Membrane Fuel Cells. J Electrochem Soc 2017;164(2):F107-14.

[35] Lee KJ, Kang JH, Nam JH. Liquid water distribution in hydrophobic gas diffusion layers with interconnect rib geometry: an invasion-percolation pore network analysis. Int J Hydrogen Energy 2014;39:6646-56.

[36] Wu R, Zhu X, Liao Q, Wang H, Ding Y-d, Li J, et al. A pore network study on water distribution in bi-layer gas diffusion media: effects of inlet boundary condition and micro-porous layer properties. Int J Hydrogen Energy 2010;35:9134-43.

[37] Eller J, Rose T, Marone F, Stampanoni M, Wokaun A, Büchi FN. Progress in in situ X-ray tomographic microscopy of liquid water in gas diffusion layers of PEFC. J Electrochem Soc 2011;158(8):B963-70.

[38] Schmittinger W, Vahidi A. A review of the main parameters influencing long-term performance and durability of PEM fuel cells. J Power Sources 2008;180:1-14.

[39] De Bruijn FA, Dam VAT, Janssen GJM. Durability and degradation issues of PEM fuel cell components. Fuel cells 2008;8:3-22.

[40] Nandjou F, Poirot-Crouvezier JP, Chandesris M, Blachot JF, Bonnaud C, Bultel Y. Impact of heat and water management on proton exchange membrane fuel cells degradation in automotive application. J Power Sources 2016;326(15):182-92. 